\title{
Augmenting Collaborative MOOC Video Viewing with Synchronized Textbook
}

\author{
Nan Li, Łukasz Kidziński and Pierre Dillenbourg \\ CHILI, EPFL, Switzerland \\ \{nan.li,lukasz.kidzinski, pierre.dillenbourg\}@epfl.ch
}

\begin{abstract}
We designed BOOC, an application that synchronizes textbook content with MOOC (Massive Open Online Courses) videos. The application leverages a tablet display split into two views to present lecture videos and textbook content simultaneously. The display of the book serves as peripheral contextual help for video viewing activities. A five-week user study with 6 groups of MOOC students in a blended on-campus course was conducted. Our study in this paper reports how textbooks are used in authentic MOOC study groups and further explores the effects of the book-mapping feature of the BOOC player in enhancing the collaborative MOOC learning experiences.
\end{abstract}

Keywords. MOOC, peripheral display, contextual help, collaborative learning

\section{Introduction}

Massive Open Online Courses (MOOCs) are in recent years growing in popularity. Popular platforms such as Coursera and edX typically replicate traditional classroom pedagogy online, featuring with video lectures, quizzes, tutorials, discussion forums and wikis. Among these learning components, lecture videos play a central role in MOOC learning; the forums provide peer-to-peer support analogous to that in the classroom, and other components mainly serve as supporting resources. Note that textbooks are not essential for MOOCs. Their role as references is perhaps partially displaced by the lecture videos. This does not mean textbooks are not useful in MOOC learning. In a MOOC taught with a companion textbook, Belanger et. al [1] found that the students often spontaneously identified related content in the textbook, then shared and discussed them in the forum. This finding does not only exhibit that textbooks still function as potentially effective supporting materials for MOOCs, but also indicates the potential needs for providing the students with supervised book-tovideo references.

Apart from textbooks, study groups, which is a common collaborative learning setting at schools and universities, are also suppressed by MOOCs, particularly by its massive and distributed nature. Compared to online forums and online groups, collocated study groups allow more intimate discussions. In our recent work [4], we have shown that students are enthusiastic about studying MOOC in face-to-face groups. We also explored how students studied in different group arrangements, i.e. watching 
videos on individual displays or on a shared display. The key question to be addressed by this paper is, however, about a combinational usage of study group and textbook. We are interested in how the students use textbooks in their groups. Particularly, when referenced textbook content is linked to the videos, how does it affect the students' collaborative learning behaviors?

Textbooks are known to offer more comprehensive learning materials. When the books are linked to videos, the support becomes contextual help. Such help has been recognized as effective means for learning graphical interfaces [2]. In fact, most of the contextual help literature dealt with step-by-step web tutorials, and only several investigated video-based tutorials. Pause-and-Play [5] proposed a method to detect taskperforming events in the video and link them with user actions in the target application as the user tried to imitate the procedure. This method avoids manually switching between the user context and the online tutorial. ToolClips [7] embedded video tutorials as contextual assistance for tool functionality understanding in a software application; FollowUs [6] demonstrated that video tutorials could be enhanced by multiple demonstrations of tutorial videos from other community members. These projects endeavored to create links between graphical interface to be learned and video tutorials. Practicing the software is the main activity that renders the context whereas the videos are seen as external helps. However, situations are poles apart in the MOOC scenario, where video viewing is the central activity. Additionally, video viewing is more passive and engaging, capturing most of the learners' attention.

In this paper, we evaluate $\mathrm{BOOC}$, a $\mathrm{MOOC}$ video player that synchronizes lecture videos with relevant textbook pages in an authentic study group setting. The book pages are displayed in the periphery to minimize distractions. The paper contributes in understanding the roles of textbooks, especially when used as peripheral contextual help in MOOC study groups. We reveal how the students leverage the usage of books and peer discussion to study MOOC in groups.

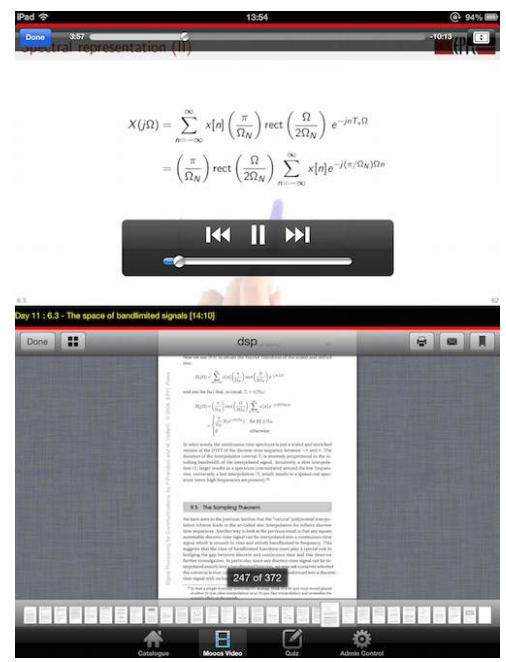

Fig. 1. The BOOC Player 


\section{The BOOC User Interface}

As shown in Fig. 1, the BOOC user interface is separated into two parts, a video controller plays the lecture video and a PDF controller manages page navigations. Each controller has a horizontal status indicator, both of which are initially red, indicating the contents are synchronized. Double clicking on the PDF controller toggles the synchronization. Desynchronized PDF controller will a have green indicator, which contrasts the other one in red, suggesting asynchronicity.

The two controllers are synchronized by default. If the video content is beyond the textbook, the PDF controller is greyed out, indicating that no textbook pages are relevant at the moment. In case multiple pages are related to the same video segment, the most relevant one is presented. Others have only their page numbers shown as yellow text in the middle of the screen. Students can navigate to those pages at their own effort, if they wish.

Videos and books are dually mapped. Users can also navigate through the digital book to get the corresponding video explanations, if available. In case of multiple mappings, the system pops out a list of other relevant videos for selection.
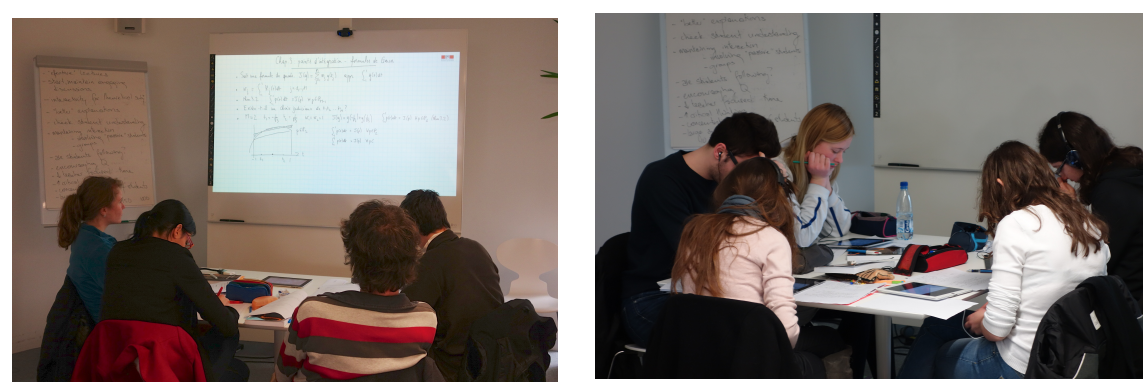

Fig. 2. CC (left) and DD (right) Group Conditions

\section{User Study}

Our study was based on an undergraduate-level MOOC offered by our university at Coursera, Numeric Analysis. The on-campus version of the course was arranged as 7week flipped teaching with MOOC plus 8-week traditional teaching. In the flipped teaching period, the students were required to watch videos and solve quizzes at home. Classroom sessions were reserved for exercises and advanced tutorials. We recruited 25 volunteered on-campus students ( 8 females/ 17 males) from the course. Each subject was compensated $150 \mathrm{SFr}$. together with a print companion textbook for participating 5 weekly study sessions. Since it is common for students to study with familiar fellow students, we allow them to spontaneously form 6 groups by themselves.

Each group met weekly around a table to study together. Activities typically included watching videos and solving quizzes. Half of the groups shared a tablet that was connected to a beamer for video viewing, and we call this condition CC (Central- 
ized display Centralized control, see Fig. 2 left). Students from the rest groups watched videos on her own tablet (borrowed from us) independently with earphones, and this condition is referred to as DD (Distributed display Distributed control, see Fig. 2 right). All the groups were composed of 4 students, except one DD group has 5 participants. We believe the two group conditions both naturally exist in real life, so we would like to understand how the groups study and use books accordingly. In other words, in spite of the group conditions, the study is more of an exploration rather than a comparison of experimental designs.

In addition, we did not instruct the students how to watch videos or collaborate. They were encouraged to behave naturally. However, we manipulated the forms of the video player. The subjects watched the videos for the first three weeks with a normal video player (without PDF); the BOOC player with book mappings was used only for the last two weeks. To our knowledge, there was no reliable automatic way to map book content with videos precisely, so we made it manually with the help of a designated teaching assistant of the course. Each week's videos (less than 10) took around one hour to finish the mapping.

For the data collection, we have video interaction logs obtained from the tablet video player. We also videotaped the study sessions so as to analyze the book usage and speech from the recordings. Two cameras were employed to capture both the front and rear view of the group interactions. In addition, there were weekly recurring post-experiment questionnaires with repeated questions regarding group collaboration, quality of discussion as well as book usage. Each session was followed by a semi-structured interview so as to obtain deeper insights of their learning experiences.

\section{$4 \quad$ Results}

The event logs recorded the interactions on the BOOC player, but print book interactions as well as collective interactions involving multiple subjects need to be manually coded from the video recordings. One of the authors coded 440 book interactions found from video recordings of 30 study sessions (2-3 hours each), and identified the following interactions modes:

- Browse. Turning the print book rapidly to look for relevant pages

- Glimpse. Glancing at the book to follow the video (mostly) or quiz. Such interactions typically lasted shortly, and the students' eyes usually quickly moved back-and-forth between the book and the video / quiz sheets

- Read. Resting the eyes on the print book page for longer time to read text

- Turn. Turning pages on the PDF book.

- Scroll. Scrolling the PDF book to view different parts of the page.

- Zoom. Zooming in / out to see details in the PDF book

- Talk. Talking to other members with the book, sometimes with deictic gestures

Among these modes, Browse, Glimpse and Read are individual interactions on the print book; Turn, Scroll and Zoom are associated with the embedded PDF in the BOOC player. An interaction becomes collective if it is performed with concious 
awareness of mutiple group members, e.g. when they have shared interest in the book or discuss the book. Talk is a collective verbal interaction by definition. The presented interaction modes can be easily identified from the videos with the help of both front view and rear view recordings. In principle Glimpse and Read also apply to the PDF, but it is difficult to tell from the video recordings without eye tracking, since the videos and PDF reside in parallel.

\subsection{Textbook Usage in Pre-BOOC Sessions}

In the first three weeks, the students watched lecture videos on normal tablet video players, and their companion textbooks were all placed on the table. Each week, a new sequence of videos was available, and we found that the students usually watched them in order. As soon as a group finished watching a video, they often had short discussions about the just-watched video or the associated quizzes before starting the next video. This period of time is referred to as video break, as compared to video pause, which refers to the time when a video is paused during its watching session. The DD students tended to synchronously start the videos and also tried to finish the videos at the same time so as to start discussions together. More thorough discussions about this phenomenon are presented in [4]. The book was not essential for studying the course, so the usage was voluntary. Not every group had used books in every session, and our goal was not to predict the usage. Rather, our interest lies in the interaction modes when the book was used. Fig. 3 illustrates the frequency of each print book interaction mode of the DD and CC groups in the first three weeks. We find that the time period when the book interactions occurred differed significantly between CC and DD $\left(\chi^{2}(200,1)=34.98, p<.0001, \phi=0.43\right)$. The CC groups had more balanced usage of book between during the video break and video watching, while the DD students mostly used the textbooks in video breaks. This was especially notable for the Glimpse action, which indicated the student was following the videos with textbooks in parallel. The frequency is visibly high in the CC but none in the DD. A possible explanation could be the DD students did not want to break their video watching synchronicity for using the book.

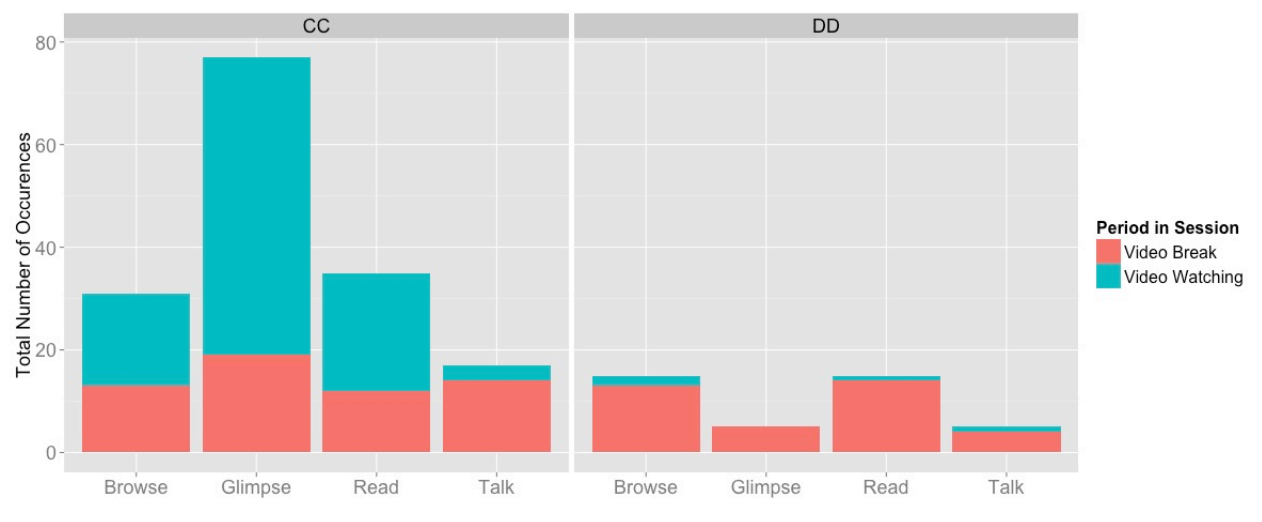

Fig. 3. Frequencies of the Print Book Interaction Modes in Pre-BOOC Sessions 
Overall there were 20 occasions where the students grabbed the book on the table and looked for relevant content while watching videos (Browse). 32.5 seconds on average were spent on each Browse. In 3 out of the 20 Browse occasions, the students failed to find the intended information on the book. Collective book interactions were only in the form of Talk with print books. Usually we found a student asked questions to others with the book or read aloud the book to the group. Talk interactions account for respectively $10.6 \%$ and $12.5 \%$ in CC and DD and mostly (82.3\% and $80.0 \%$ ) happened during video break.

During the semi-structured interviews, we tried to understand why some of the students did not use the book during the interviews. Main reasons include (1) they were afraid of loosing time in looking for information in the book (2) they did not know exactly what is not clear while watching videos (3) the lectures were easy, so the videos are sufficient for learning (4) they prefer to ask the groups, which usually solved their problems. Feedbacks (3-4) reflect more of contextual constraints in the collaborative MOOC learning setup, where the students' doubts about video content depend on the course itself. They may prefer to address the problems through discussions in the group rather than the book. Feedbacks (1-2) as well as the observations presented before confirm the potential needs for peripherally displaying book references, so that the students can quick judge the usefulness of book content.

\subsection{Textbook Usage in BOOC Sessions}

We deployed the BOOC player with book mappings in the last two weeks of the study, but students were still asked to take out their print textbooks during the study sessions for potential usage. In fact, only one student had used the print book in the last two weeks, because he favored the tangibility of the print book. Still, he used the PDF mapping in BOOC to help quickly navigate to the pages.

To analyze the usage of the peripherally displayed synchronized PDF, we first summarize the usage scenarios collected from the questionnaire as below: (1) Extended Knowledge: when they saw another explanation of a concept with detailed theorems and examples (2) Alternative Presentation: when the teacher was talking too fast or the videos were not visibly clear (3) Information Confirmation: when the students had doubts about certain concepts and need to confirm their understanding (4) External Help: when none of the group members knew the answer or when they were arguing about certain concepts. Among these usage scenarios, the advantage of the peripheral design is especially notable in (1), where the students had no explicit needs of help - They actually discovered helpful information in the peripheral display of the book with serendipity. 


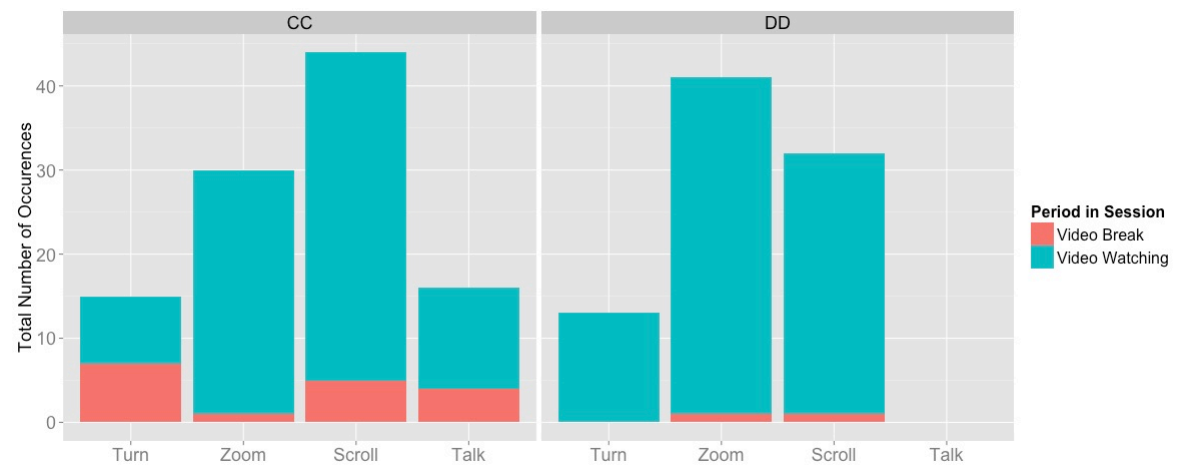

Fig. 4. Frequencies of PDF Interaction Modes in BOOC Sessions

Fig. 4 depicts the frequency of book interactions by mode with BOOC interface. It is not surprising to see that in both conditions, the interactions predominantly happened during video watching rather than during video break due to the book-mapping feature. What is more interesting is that the DD students used the book frequently during video watching, which seldom happened before. A possible explanation could be the synchronized PDF increased the visibility and accessibility of the potentially useful information in the book. As a result, the students were offered better opportunities to address their situational needs without the fear of loosing synchronicity.

For the students in the $\mathrm{CC}$ condition, the most notable change after the introduction of BOOC is the increased frequency of collective activities, especially during video watching. The proportion of collective activities doubles to $20.9 \%$. If we count only Talk interaction, the proportion is $15.2 \%$, with $75 \%$ happened during video watching, as compared to $17.7 \%$ in the pre-BOOC sessions. The reason behind the increments, we believe, is that the shared display of synchronized book content increased shared attention, so that the students could have more chances to collaborate with the book. One may wonder why the increment of book discussion matters. In fact, when the quality of discussion (5-point Likert scale ratings obtained from weekly questionnaires) in the CC groups was predicted, it was found that the frequency of Talk interactions during video watching $(\beta=0.24, \mathrm{p}<.05)$, the proportion of speech during video break $(\beta=5.58, \mathrm{p}<.001)$ were significant predictors. The statistics were computed from a mixed-effect multiple-regression model where the students nested in groups were modeled as random effects. The overall model fit in terms of R-squared was 0.48 , and the marginal R-squared contributed by the fixed effects was 0.21 . Significance could also be obtained if we fitted in the model with the frequency of all collective interactions or the duration of Talk interaction, instead of Talk frequency. This finding indicates that more discussions with book or collective book usage in general may increase discussion quality.

Remember that when we designed the BOOC interface, we enabled dual mapping between the PDF and the video. During the experiment, linking from book pages to videos were never intended, since watching videos was their main activity, not read- 
ing books. The students were sometimes annoyed due to abrupt video changes when they accidentally swiped the PDF to a page with a different video mapping. We argue dual mapping might be useful when used at home than in time-bounded group study sessions, where the students' activities are much centered on watching videos.

\section{Conclusion}

In this paper, we presented how textbooks were used in two common formats of authentic MOOC study groups. Particularly, we summarized the students' feedback on why print textbooks were not widely accepted and when the BOOC interface, which was essentially a peripheral contextual help display, was considered useful in the group study sessions. We also delivered an empirical categorization of book interaction modes during study group sessions. These qualitative findings increased our understanding of the role of the textbooks in collaborative MOOC learning setup.

In addition, we discussed how the book usage was changed since the introduction of BOOC player in a quantitative way. Most importantly, we found the BOOC player increased collective book usage for the CC groups, which in turn enhanced the students' discussion quality significantly. These findings further revealed the effectiveness of the peripheral book display as it increased mutual awareness of contextual information. Even in the DD condition, the display worked in the sense that it provided situational help to the students without losing much synchronicity, which is essential in the DD style study groups [4]. Future work includes replicating similar interfaces to the more dynamic online platforms, and contextual helps other than textbook may be employed to facilitate students' learning as well.

\section{References}

1. Belanger, Y., Thornton, J., Barr, R. C.: Bioelectricity: A quantitative approach--Duke University's first MOOC. Duke Univeristy (2013)

2. Chilana, P. K., Ko, A. J., Wobbrock, J. O.: LemonAid: selection-based crowdsourced contextual help for web applications. In: Proceedings of CHI'12. ACM (2012)

3. Chi, P. Y., Ahn, S., Ren, A., Dontcheva, M., Li, W., Hartmann, B.: MixT: automatic generation of step-by-step mixed media tutorials. In: Proceedings of UIST'12. ACM (2012)

4. Li, N., Verma, H., Skevi, A., Zufferey, G., Blom, J., Dillenbourg, P.: Watching MOOCs together: investigating co-located MOOC study groups. Distance Education, 35, 217-233 (2014)

5. Pongnumkul, S., Dontcheva, M., Li, W., Wang, J., Bourdev, L., Avidan, S., Cohen, M. F.: Pause-and-play: automatically linking screencast video tutorials with applications. In: Proceedings of UIST'11. ACM (2011)

6. Lafreniere, B., Grossman, T., Fitzmaurice, G.: Community enhanced tutorials: improving tutorials with multiple demonstrations. In: Proceedings of the CHI'13. ACM (2013)

7. Grossman, T., Fitzmaurice, G.: ToolClips: an investigation of contextual video assistance for functionality understanding. In: Proceedings of CHI'10.ACM (2010) 